\title{
EL $=\underline{=}=\underline{=}=\underline{=}=\underline{=}=\underline{=}=\underline{=}=\stackrel{C U E R P O S}{=}$
}

C. Simo

\$1. - Ecuaciones $y$ definicion del probiema. Integrales primeras: Teoremas de Bruns, painleve y Poincaré.

Desde el punto de vista matematico consideraremos el problema de $n$ cuerpos como el de $n$ masas no nulas, puntuales, sometiöas a la ley de la atraccion universal de Newton. Si $x_{i}$ es la posicion del i-esimo cuerpo respecto al c.d.n. $y m_{i}$ su masa, se tiene

$$
\ddot{x}_{i}=\sum_{i \neq j=1}^{n} m_{j} \frac{x_{j}-x_{i}}{\left|x_{j}-x_{i j}\right|^{3}} \quad i=1 \div n
$$

como sistera de ecuaciones diferenciales que rige el movimiento de los in cuerpos. (a)

Desde el punto de vista ffsico este es un modelo idealizado. Por un lado los cuerpos celestes no son puntuales. Por otro Ias Iuerzas en juego pueōen ser de otro tipo distinto ai $1 / r^{2}$ newtoniano (sin embargo muchos de los resultados de la teorfa siguen siendo validos para otras fuerzas, por ejemplo del tipo $1 / r^{*}$ $q \in[1,3]$, o exigiendo solo comportamiento $1 / r^{2}$ para distancias grandes). Finalmente (1) implica una hipotesis conservativa. Las pérdidas de energía por efecto de mareas, por ejemplo, no se tie nen en cuenta. Volveremos a este punto mas adelante (en $\$ 5$ ).

otro aspecto a tener en cuenta es que condiciones iniciales desprẹciables matematicamente, por ejempio un conjunto de medida nuła en el espacio fasico, pueden ser fundamentales fisicamente. Supongamos que el inicio del cosmos fuera debido a una explosion. Se tendría entonces momento angular total nulo, lo que restringe el movimiento a una variedad de medida de tubesgue nula.

A falta de una mejor comprension de las leyes fisicas que rigen el movimiento ae los astros tomaremos (I) como modelo. Este

(a) Se supondra en lo que sigue que la constante de Newton vale 1. 
es un sigtema de $6 \mathrm{n}$ ecuaciones de primer orden. Son clásicas las in tegrales primeras del centro de masas y de su velocidad, del momento angular $c=\sum_{i} m_{i} x_{i} \wedge \dot{x}_{i} y$ de la energia $h=\sum_{i} \frac{1}{2} m_{i} \dot{x}_{i}$ $-\sum_{1 \leq i \leq j \leq n} \frac{m_{i} m_{j}}{\left|x_{i}-x_{j}\right|}$. En total se dispone de 10 integrales primeras. Ademas, debido a que la acción de $s^{2}$ en el espacio fásico transforma toda solucion de (1) en otra solución (eliminación del nodo) y a que es posible eliminar el tiempo como variable independiente, se tiene una reAucción a un sistema de $6_{n}-12$ ecuaciones. Así el problema de 2 cuerpos queda resuelto $y$ el de 3 , reducido a 69 orden. Tal reduccion puede verse en whittaker.

Es natural preguntarse por la existencia de más integrales prime ras de (1). Por el momento no se conoce ninguna otra (con caracter global; vease losco para formas invariantes) y las experiencias numé ricas efectuadas parecen indicar que no existen más integrales primeras (ver $\$ 5)$.

Resultados clásicos en esta dirección son los célebres

Teorema (Brung 1887): Toda integral primera de (1) algebraica en $x, \dot{x}$, es función algebraica de las integrales clásicas.

Teorema (Painlevé 1896-1898) : Toda integral primera de (1) algebraica en $\dot{x}$ es funcion algebraica de las clasicas.

Teorema (Poincare 1889): 'sea $F=F_{0}+\mu F_{1}+\mu^{2} F_{2}+\ldots$ el hamiltoniano del problema de $n$ cuerpos desarrollado en potencias de un pequeño pará metro u (por ejemplo, la masa de uno de los cuerpos). Suponemos F ana litico en $u, F_{0}=F_{0}(p)$ (independiente de $q$ ), $F_{i}$, funciones de $p, q$, $i>0$

$2 \pi$ - periodicas en $q$. Suponemos, ademas, que para cada cuerpo $p_{i, q_{i}}$ son variables canonicas asociadas a los elementos elfpticos. Entonces el sistema

$$
\dot{p}_{i}=\sim \partial F / \partial q i \quad \dot{q}_{i}=\partial_{F} / \partial_{\mathrm{p}} \text { no tiene más integrales primeras }
$$

analiticas en $\mu, p, q$ y $2 \pi$-peribdicas en $q$ que las clásicas.

Este teorema no es excesivamente sorprendente ya que se sabe (Mosed 
que para cierto conjunto denso de sistemas hamiltonianos no existen integrales primeras de clase $\xi^{1}$ distintas del hamiltoniano.

\$2.- 2 cuerpos: Solucion; efemerides; determinacion de orbitas. Coligiones: Regalarizacion LC y KS. Espacio de orbitas. Transferencia.

El problema más sencilio. Gnico resuelto $y$ el que sirve de base a las técnicas de perturbaciones y a la mayoría de problemas de la Astrodinamica es ei de 2 cuerpos. Para estudiar el movimiento (Keple riano) de-uno de los cuerpos respecto al c.d.m comin (u, homot.éticamente, respecto al otro cuexpo) se introducen 2 ángulos:I (inclinación) y $O$ (argumento del nodo) para fijar la posición del plano de la $6 r b i$ ta respecto a un sistema de referencia dado. La orbita en dicho plano es una conica de semieje a y ex centricidad e, con un foco en el c. d.m. - Se define el ángulo w (argu mento del periastro) como el que forman la direccion del nodo y la del punto de la orbita más cercano al c.o.ill. (periastro).

Los elementos $a, e, I, B, w, \pi_{0}$, se llaman Keplerianos. El sexto $\pi_{0} 0$ anomalía media en la época está de

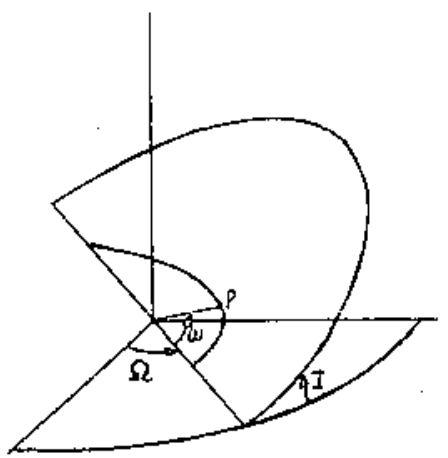
finido modulo $2 \pi$ y varía linealme-tecon $t$. Los demás son constantes. La posición en la brbita se determina mediante el ángulo v, anomalía verdadera, formada por el periastro $y$ la posicion actual del astro. Este se relaciona mediante $t_{g} \frac{v}{2}=\sqrt{\frac{1+e}{1-e}} t g t / 2$ con la llamada anomalia excéntrica $\mathrm{E}$, angulo auxiliar, que a su vez se relaciona con el tiempo mediante $M=M_{O}+t a^{-1 / 3}=E+e \sin E(\bmod 2 \pi)$.

Esta ecuación se conoce como ecuacion de Kepler. Su resolucion impulso en parte la contribución de cauchy a la teorfa de la variable compleja. Una expresion de E-M como serie en sin KT con coeficientes 
funciones de e fue dada por Lagrange en 1769. Los coeficientes re sultan ser funciones de Bessel, cuyo estudio sistemático se abor daría por primera vez 55 años más tarde.

El proceso de determinar la posicion de un astro del que se co nocen los elementos se llama calculo de efenérides. Sin embargo, para el cáculo efectivo de la posicion deberemos tener en cuenta la presencia de perturbaciones que alteran el problema de 2 cuerpos.

El problema inverso: conocida la posicion del astro calcular sus elementos, se conoce como determinacion de orbitas. Debido a la dificultad de conocer $x y \dot{x}$ en un instante dado, dicho calculo se convierte en un problema de valores en la frontera.

Es tipica la determinación de la 6rbita de un asteroide o un cometa mediante observaciones angulares, esto es: para cada instan te de tiempo se conoce en qué direccion está respecto al observador, pero no se saben nj su distancia ni su velocidad.

Los problemas motivados por la disponibilidad de un elevado numero de observaciones no compatibles debido a los errores de ob servacion llevaron a Gauss a desarrollar en 1801 el método de minimos cuadrados.

Otro ejemplo tipico de determinacion de 6rbitas en el problema de 2 cuerpos es el del rendez-vous o interceptacion: los datos de que se parte son $t_{1}, x_{1}, t_{2} y \bar{x}_{2}$.

Notable interés presenta el estudio de las 6rbitas de colision (estofes, en que los dos cuerpos chocan). En el espacio fásico (6 dimensional) lienan una variedad 4 dimensional, por loque su medida es nula. Destaquemos que (1) deja de tener sentido si $x_{1}=x_{2}$ : la trayectoria ha liegado a una singularidad.

Es natural preguntarse si es posible encontrar un cambio de vạ riables $x\left\{\begin{array}{l}y \\ 0\end{array}\right\}$ t que permita regularizar (1). esto es, transformala en una ecuación sin singularidades, $y$ en caso afirmativo inter- 
pretar fisicamente el resultado.

Para movimiento en $R^{2}$ la respuesta fue dada en 1906 por LeviCivita. La transformacion

$$
L(z) z=\left(\begin{array}{cc}
z^{1} & -z^{2} \\
z^{2} & z^{1}
\end{array}\right)\left(\begin{array}{l}
z^{1} \\
z^{2}
\end{array}\right)=\left(\begin{array}{l}
x^{1} \\
x^{2}
\end{array}\right), d s=|x| d t
$$

que introduce un nueva variable espacial $\mathrm{z}$ ( la raíz cuadrada de $x$ considerados $x, z \in \mathbb{C}$ ) $y$ una nueva temporal, s, transforman (1) en $z^{\prime \prime}+\frac{h}{2} z=0(2)$, donde $'=\frac{d}{d s}$ y h es energía total. Se obser va de paso que (2) es la ecuacion de un movimiento armónico.

Se busco en vano una matriz en $\mathrm{R}^{3}$ que generalizara la matriz de Levi-Civita L(z), o sea, cuya primera columna fuera z y las demás fueran ortogonales entre st y a z. Esta imposibilidad es de bida al teorema de Poincare de no existencia de campos vectoriales continuos en $s^{2}$.

Sin embargo sî existen dichos campos (que además engendran el e. tangente) en $\mathrm{s}^{3}$. (Un célebre resultado establece que $\mathrm{s}^{1}, \mathrm{~s}^{3}, \mathrm{~s}^{7}$ son las unicas esferas con esa propiedad).

En $R^{4}$ se sustituye $L(z)$ por

$$
\bar{L}(z)=\left(\begin{array}{ccc}
z^{1}-z^{2}-z^{3} & z^{4} \\
z^{2} z^{1}-z^{4}-z^{3} \\
z^{3} z^{4} & z^{1} & z^{2} \\
z^{4}-z^{3} & z^{2}-z^{1}
\end{array}\right) \text {. La aplicacion } \begin{aligned}
& R^{4} \rightarrow R^{4} \\
& z
\end{aligned}
$$

fue dada por Hopf en 1931 y resucitada por Kustaanheimo y Stiefel en 1965 que la aplicaron a la regularizacion. Se sumerge $R^{3}$ en $R^{4}$ haciendo $x^{4}=0$. El cambio $L(z) z=x, d s=|x|$ dt convierte (1) en (2). La fibra de cada punto de $\mathrm{R}^{3}$ por la aplicacion Ks es una circunferencia de centro el origen y radio $\sqrt{|x|}$. Tanto en el ca so LC como en el KS la regularizacion equivale ffsicamente a una co lision elástica: El movimiento Kepleriano se prolonga después de lạ colision conservando energla y cambkando de signo la cantidad de mo! vimiento.

Otra pregunta que podemos formularnos acerca del problema de dos cuerpos es la de qué estructura topologica puede darse al conjun-i 
to de las orbitas $\theta$. Este puede obtenerse del espacio fasico identi ficando todos los puntos de una misma orbita o bien, equivalentemen te, definiendo una distancia entre orbitag, ya sea a partir de las integrales primeras o geométricamente, extendiendo adecuadamente la metrica de Hausdorff a conjuntos no acotados. para que se tenga un espacio separado deben identificarse la brbita de llegada a una colisión con la correspondiente de salida (Souriau). Moser prob6 (1970) que para $h<0$ fijado, el conjunto de orbitas es homeomorfo a $s^{2} \times s^{2}$ (y que la subv. del espacio de las fases corresponaiente ea $T_{u} S^{3}$ ). Generalizando este resultado se tiene

Teorema (Sim6 1974) El conjunto de las 6rbitas del problema de 2 cuerpos es homeomorfo a $s^{2} \times s^{2} \times R-\left\{(a,-a, b) \mid a \in s^{2}, h \geq 0\right\}$. Este resultado implica la no existencia de "elementos" sin singularidades en el problema de 2 cuerpos, pues una carta es insuficiente. otra consecuencia debida al teorema de poincaré de no existencia de campos continuos en $\mathrm{S}^{2}$ es que no existe ninguna aplicación continua $\theta \rightarrow \cdot R^{3}$ que asigne a cada brbita un punto de la misma. Esto es, ningun elemento del tipo Mo puede ser asignado de.forma continua a una orbita.

Finalmente un problema que no se halla en la naturaleza pero aparece en astrodinamica es el de transferencia optimal de orbitas. Se trata de pasar de una orbita Kepleriana a otra de manera que el consumo de energía en dicho paso sea minimo. El problema está resuel to en el caso de que ambas 6rbitas sean coplanarias, pero no en el caso general. Además existen casos en los que la transferencia 6pti ma desde el punto de vista energético requiere tiempo infinito (las llamadas transferencias por el infinito, a traves de 2 orbitas intermedias).

3. - Tres cuerpos: Pequeno T. de Sundman. Teorema de Sundman. Soluciones conocidas: soluciones periodicas. Posiciones de equilibrio relativo. Interés en el cálculo de variedades invariantes. 
Bl paso de 2 a 3 cuerpos representa la aparicion de multitud de diferencias. Podemos decir que en el problema general (masas cualesquiera) el conocimiento de soluciones del problema de 2 cuerpos no representa ninguna aportacion.

Un primer teorema que se aplica a un no cualquiera de cuer pos es llamado t. del colapso total o pequeno t. de sundman: condi ción necesaria para que los n cuerpos colisionen es que el momen to angular total sea nulo. Por tanto si $c \neq 0$ y $n=3$ solo puede haber colisiones dobles. Sundman probo que la presencia del ter cer cuexpo no es obstaculo para que el movimiento sea regulariza ble, $y$ de heaho, si no se anula $c$, dio la solución al problema de 3 cuerpos.

Teorema (Sundman(1913) Si $c \neq 0$, las coordenadas cartesianas $y$ el tiempo son funciones anal1ticas de $w$ que convergen $s i|w|<1$, donde

$$
w=t h \frac{\pi s}{84}, s=\int_{t_{0}}^{t}(U+1) \text { dt } \quad \text { siendo } U \text { la energía }
$$

potencial y 6 un numero positivo funcion de las masas y de las condiciones iniciales para $t=t_{0}$. En particular el desarrollo es valido para toda $t$ real.

A pesar de que este teorema resuelve el problema de 3 cuerpos excepto en un caso de medida nula $(c=0)$, no es práctico dada la lenta convergencia de las series $y$ el hecho de que no suministra informacion del comportamiento cualitativo.

La obtencion de soluciones para intervalos de tiempo relativamente pequenos no ofrece dificultad (cea desde el punto de vista analitico o numérico. véase sin embargoflo).sin embargo si queremos pre decir algo para tiempo no acotado tendremos dificultades.

Estas desaparecen si la Grbita es periodica. En este caso el conocimiento de la misma para un tiempo finito permite conocerla para todo tiempo.

Poincare conjeturo que dada una orbita $x(t)$ de condiciones 
iniciales $\mathrm{x}_{0} . \dot{\mathrm{x}}_{0}$ para $\mathrm{t}=0 \quad \forall \varepsilon>0, \forall \mathrm{T}>0$ existen condiciones $\mathrm{x}_{0}^{*}, \dot{\mathrm{x}}_{0}^{*}$ tales que $\left|\mathrm{x}_{0}-\mathrm{x}_{0}^{*}\right|<\mathrm{c},\left|\dot{\mathrm{x}}_{0}-\dot{\mathrm{x}}_{0}^{*}\right|<\mathrm{e}$ dan una $6 \mathrm{r}-$ bita peribdica $x^{*}(t)$ de manera que $\forall t \varepsilon[0, T]\left|x(t)-x^{*}(t)\right|<e$. Esta conjetura, a la que habla que añair $h<0$ es la que ha impul sado la brsqueda de orbitas peribdicas. Actualmente no se conoce todavía la certeza o falsedad de dicha conjetura.

En el caso de que 2 de las masas fueran pequeñas lcaso planetario) Poincaré divioio las 6rbitas en 3 especies:

$$
\begin{aligned}
& \text { 1) } I=0, e=0 \\
& \text { 2) } I=0, e \text { grande } \\
& \text { 3) } I \text { grande, } e \simeq 0
\end{aligned}
$$

Ia busqueda efectiva de soluciones peribdicas del problema de 3 cuerpos, se aborda a partir del problema restringido (ver $\} 4$ ), pe ro una exploración completa dista de llevarse a cabo.

Un tipo particular de soluciones peribdicas es aquél en que se pasa de $x_{i}(0)$ a $x_{i}(t)$ mediante una homotecia $H_{t}$ funcion de $t$, idéntica para $i=1 \div \mathrm{n}$, (en particular $\mathrm{H}_{t}$ puede ser un girol. Euler (1765) obtuvo los valores de $x_{1}(0)$ en el caso en que $x_{1}, x_{2}, x_{3}$ estuvieran alineadas, obteniendo 3 soluciones y Lagrange (1772) determino las otras 2 soluciones restantes (siméricas) en las que los 3 cuerpos forman triángulo equilatero. (Naturalmente hay soluciones de tipo homotetico no peribdicas, llevando a colapso - a desintegracion).

El conjunto de condiciones iniciales que da orbitas homoteticas peribdicas es cerrado por homotecias. Cada clase se llama una posicion de equilibrio relativo (p.e.r.). Se demuestra que en una p.e.r. Ios $n$ cuexpos deben estar en un plano. El interes del conocimiento de los p.e.r. en el caso general se ha visto aumentado por el hecho de que los p.e.r. coinciden con los puntos criticos del potencial $-\sum_{1 \leq i<j \leq n} \frac{m_{i} m_{j}}{\left|x_{i}-x_{j}\right|}$ restringido a la variedad 
$\frac{1}{2} \sum_{i-1}^{n} \pi_{i}\left|x_{i}\right|^{2}=1$

Consideremos ahora la aplicación (E,J) que a cada punto deI espacio fástco del que se excluyen las colisiones le hace corres ponder energia y momento angular. Dados $h \in R, c \in R^{3}$ el conjunto $I_{h, c}=(E, J)^{-1}(h, c)$ es invariante por el flujo. EI conocimiento de $I_{\text {hc }}$ puede dar informacion sobre las brbitas con esos vaLores de energía y momento.

Smale introdujo el concepto de conjunto de bifurcacion: vaIores de $\mathrm{h}, \mathrm{c}$ donde ${ }^{I_{\mathrm{h}}}$ cambia de estructura. Gracias a regultados de Easton y cabral, se sabe que el conjunto de bifurcacion contiene a los puntos criticos.

En el caso plano del problema de n cuerpos los puntos criticos son:

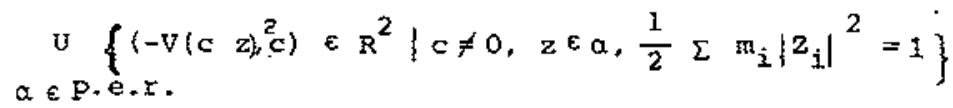

$y$ el conjunto bifurcacion es la union de los puntos criticos $\operatorname{con}$ los ejes $h, c$.

En el caso espacial los puntos criticos de (E,J) se obtienen por union deI eje $h$ con los puntos $(h, c)$ tales que (i, $|c|)$ es punto cretico deI caso plano.

El estudio del conjunto de bifurcacion en el caso espa cial debe llevarse a cabo directamente, pero en todo caso requiere conocer el de puntos criticos, $y$ este el de p.e.r.

Esto ha sido Ilevado a cabo por c. Sinb y J. LIibre. Se pue de calcular Ihc en texminos de antimágenes de $I_{c}$ conde $1_{c}(z)=-v(z)^{2} / 2 \gamma_{c}(z)$, siendo $\frac{1}{2} \Sigma m_{i}\left|z z_{i}\right|^{2}=1$, v el potencial y $Y_{c}(2)$ la energía cinetica de los cuerpos consideradoa como s6lido rígido con momento angular c. Sin entrar en detalles digamos que en el caso de masas distintas se tienen 11 estructuras de $I_{h c}$ para el caso plano y 17 en el caso espacial. 
Destaquemos que mientras en el caso plano se tienen difeomorfis mos, en el caso espacial se reducen a homeomorfismos. De hecho $I_{h c}$ no son variedades topologicas en el caso espacial: La dimension local varía.

\$4. - El problema restrinqido: Ecuaciones. Curvas de velocidad nula. Soluciones perióicas. Aplicacion de Poincare. Estabilidad. Puntos

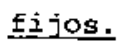

Un caso sencillo del problema de 3 cuerpos es aquel en que una de las masas es infinitesimal de manera que su influencia sobre las otras dos es despreciable. El movimiento de éstas es, pues, el de un problema de dos cuerpos. Suponöremos que éste es circular $Y$ que el 30 se mueve en el plano de los 2 primeros. Hablamos de movimiento restringico plano $y$ circular. Si usamos ejes (sinbaicos) girato rios tales que el eje $x$ contenga siempre los 2 primarios se obtiene

$$
x-z \dot{y}=\Omega_{x}, \quad \ddot{y}+2 \dot{x}=\Omega_{y}, \Omega=\frac{1}{2}\left[(1-\mu) r_{1}^{2}+u r_{2}^{2}\right]+
$$

$+\frac{1-\mu}{r_{1}}+\frac{\mu}{r_{2}}$

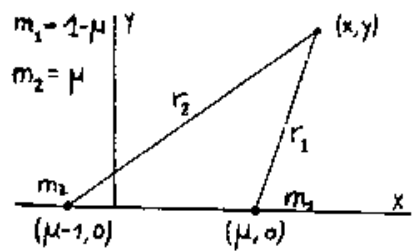

que son las ecuaciones que rigen el movimiento del cuerpo infinitesimal. Tienen una integral primera (Jacobi 1836): $\Omega-\frac{1}{2}\left(x^{2}+y^{2}\right)=c$. pijadas unas condiciones iniciales conocemos C. Haciendo $k=\hat{y}=0$ se tienen las llamadas curvas de velocidad nula que delimitan regiones (en coordenadas si-

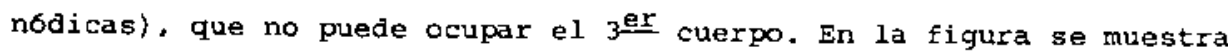
la forma de esas curvas para valores distintos de c. El movimiento solo es posible en el iaterior de las mismas Como para la luna se tiene un caso anạ logo a $c_{1}$, Esta no puede escapar de la pxoximidad de la tierra para caer hacia

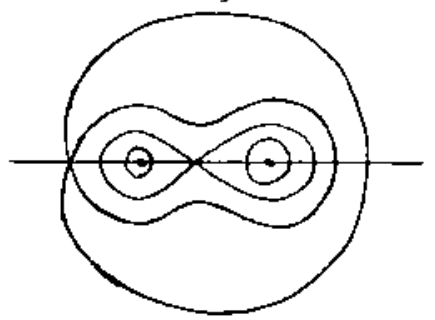
el sol (en el modelo de 3 cuerpos restringido). 
Los puntos $x, y$ en los que $\Omega$ es estacionario corresponden a po siciones de equilibrio en el sistema sinbdico y de equilibrio relativo en el sidereo. Se encuentran las ya conocidas posiciones de Euler $y$ Lagrange. El problema restringido de 3 cuerpos es suficien temente sencillo como para que saya abordado de manera exhaustiva la bosqueda de orbitas periodicas. El primer estudio sistematico se inici6 en 1900 por. Strömgren $y$ sus colaboradores con el llamado pro blema de copenhager caso en que $\mu=\frac{1}{2}$, Dada una orbita peribdica podemos buscar condiciones iniciales próximas a las de dicha orbita que nos proporcionen otra orbita peribdica. Por ejemplo, si buscamos 6rbitas periodicas simetricas, las $\mathrm{ci}$. pueden ser $\mathrm{x}_{0}, \mathrm{y}_{0}=0$, $\dot{x}_{0}=0, \dot{y}_{0}, 0$ equivalentemente $x_{0}, c_{0}$. Si la 6rbita periodica puede prolongarse (curva en el espacio, $x, c$, por ejemplo), hablatmos de familia de orbitas periodicas. (Para sistemas hamiltonianos si $\gamma$ es peribaica $y$ es una aplicacion de poincare sobre una variedad $2 n-2$ dimensional $\Sigma$ transversal a $\gamma$ contenida en la variedad de energía constante a la que pertenece $\gamma$. entonces esta orbita se ha lla en una familia I -paxamétrica de orbjtas peribdicas si 1 no es valor propio de De $(m)$ m matriz de monoaromía, siendo m $=\gamma f(\mathbf{i}$ ). (Véase Robinson).

En el caso de Copenhagen se hallaron 13 familias de orbitas periodicas, algunas de elias con varias subclases. Posteriormente (Hénon, Bartlett) se han hallado10 familias mág de orbitas periodicas simetricas, se han encontrado familias no simétricas y se han estudiado Ios casos $\mu \neq 1 / 2$.

Una herramienta basica para la obtencion de orbitas peribaicas es la aplicación de Poincare. Sobre $\mathrm{H}=$ cte., corternos el flujo trarb versalmente por una vajedad $\Sigma$ So define la aplicación de poincaré $\theta$ (quizá sobre un subconjunto de $\Sigma$ ) como la aplicación que a un punto m de $\Sigma$ le hace corresponder la proxima interseccion $\theta(\mathrm{m})$ de $\Sigma$ con el flujo pasando por $\mathrm{m}$. Es claro que $\theta(\mathrm{m})=\mathrm{m}$ es la condición de $\underline{\underline{x}}$ bita periodica (simple), 0 , en general $\theta^{k}(m)=m$. 
La aplicación ae Poincaré es simpléctica (conserva la forma dp $\wedge d q$ de $\Sigma) ~ y$ en particular preserva medida. Los teoremas de pun to fijo pueden servir para garantizar existencia de orbitas periodi cas (pero téngáse en cuenta que por preservar volumen no pueden aplicarse teoremas de contraccion).

Una primera indicacion de Ia estabilidad (orbital) de la orbita periodica la suministra el analisis del espectro de DQ. Si existen valores propios reales el punto es hiperbolico en lo que hace referencia a la parte lineal (y también a la nolineal). En ca so contrario es elfptico (linealmente) y la estabilidad frente a perturbaciones no lineales puede obtenerse en ciertos casos de la Teoria KAM (ver $§ 5)$.

$\oint 5 .-$ Totalidad de soluciones en el problema restringido: Integrabilidad. Teorema de Liouville-Arnol'd. Teorema KAM. Movimientos quasiperi6dicos. Aplicaciones: Teorema de Leontovich, Sistema solar. Difusion de Arnol'd.

Hénon ha continuado la exploración numérica en el caso del pro blema de copenhagen mediante aplicacion de Poincaré respecto al pla no $x, \dot{x}$, tratando todo tipo de orbitas ( $y$ no solo las periodicas). los resultados son muy instructivos.

Para valores $c$ grandes $(>4.5$ ) las sucesivas imagenes de $x, \dot{x}$ por $\theta$ parecen llenar una curva cerrada de un modo denso correspondiendo a trayectorias quasi peri6dicas.

Sin embargo para numero de rotacion racional (no de rotacion=fracción de vuelta girada alrededor de un punto fijo en cada aplica ci6n de $\theta$, en promedio), aparecen islas que envuelven puntos elipticos $y$ que quedan separadas por puntos hiperb6licos. A medi

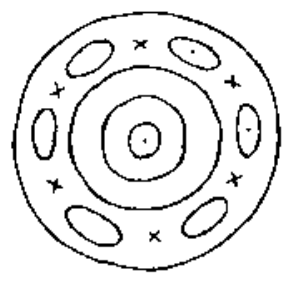


da que nos acercamos al punto fijo de $\theta$ (correspondiente a la orbita peri6dica) la anchura de las islas ( $y$ de las zonas hiperb61icas) disminuye siendo no apreciable.

Para valores menores de $C$ aparecen regiones en las que las sucesivas imágenes de $(x, \dot{x})$ por $\theta$ parecen aleatc :as $y$, en cierto modo lle nan todo el posible espacio de variación de $(x, \dot{x})$ formando 10 que se llama 'un mar ergodico en el que permanecen islag integrables".

Si un sistema hamiltoniano posee $n$ integrales primeras independientes $F_{i}$ en involucion, el teorema de Liouville-Arnol'd asegura que si $F_{i}=$ cte. $i=1 \div n$ define una variedad compacta, ésta es un tero n-dimensional (en el caso no compacto será $\left(s^{1}\right)^{k} \times$ $\times R^{n-k}$, en el que el flujo viene dado por $\dot{q}_{i}=w_{i}=c t e$.

Al cortar dicho toro por $\Sigma$ aparece esta varjedad foliada por curvas invariantes. Esta es la caracteristica de sistemas integrables. Por este motivo la presunta aparicion de esas curvas ha motivado la bisqueda de una $2^{a}$ integral (la $1^{a}$ es la de Jacobi). En problemas más generales, esto es, con mayor no de grados de libertad se habla de la búsqueda de la $3^{\text {à }}$ integral (siendo las 2 primeras Ias de energfa $y$ momento). Es slaro que esta búsque da es formal ya que la apaxicion de puntos elfpticos e hiperbó 1 icos de $\theta^{k}$ entre curvas invariantes de $\theta$ es general. En este aspecto una aproximacion mediante la forma normal de Birkhoff es Gtil.

En el extremo opuesto de los sistemas integrables están los ergodicos.ta aplicación $\theta$ es ergodica si para toda $f \in I^{I}(\Sigma)$, la media de $f$ sobre $\Sigma$ (supuesto $\Sigma$ compacta) es igual a la media "tem poral" sobre la trayectoria por $\theta$. Esto es: $\forall \times \in \Sigma$

$$
\lim _{N \rightarrow \infty} \frac{1}{N} \sum_{k=0}^{N} f\left(\theta^{k} x\right)=\frac{I}{\mu(\Sigma)} \int_{\Sigma} f d .
$$


Se tiene de inmediato que si $\theta$ es ergodica todo subconjun to propio de $\Sigma$ invariante por $\theta$ tiene medida nula.

En Ia practica parece que todo sistema hamiltoniano tiene parte de "componente integrable" y parte de "componente ergo dica". Sin embargo no esta claro como abordar el problema.

Si un sistema hamiltoniano $H$ esta cerca de un sistema in tegrable $\mathrm{H}_{0}: \mathrm{H}=\mathrm{H}_{0}+\varepsilon \mathrm{H}_{1}$, podemos preguntarnos si retiene algo de las caracterssticas de integrabilidad.

La respuesta la da el teorema de Kolmogoroff-Arnol'd-Moser (1954-1962-1963) probado por Arnol'd por el caso analftico. Moser para el caso $\varphi^{333} \quad y$ Iuego por Rumann para $b^{5}$ (se sabe que para $\mathscr{b}^{t}$ es falso), que asegura (tanto para sistemas hamiltonianos como para aplicaciones globalmente canonicas) que si las perturbaciones son suficientemente peque has $y$ se cumple una condición de no degeneración de $\mathrm{H}_{0}$ (xela tiva a su jacobiano $y$ hessiano $)$, para la mayor. parte de los toros invariantes estos sufren una deformacion pero no desapa recen. Esta "mayor parte" esta formada por aquellos toros pa ra los que $\forall k \in z^{n}, \forall k_{0} \in z \quad f(k, w)-\left.k_{0}\left|>c_{w} /\right| k\right|^{v}$ donde $C_{\text {tw }}$ es una constante que depende de $w, v \geq n+2$ es un entero adecuado $y \quad|k|=\Sigma\left|k_{i}\right|$. Observemos que en dimension 1 hay que eliminar como postbles valores de w los racionales y los nes de fioville.

Sobre los toros invariantes el flujo es el de un movimiento quasi peribaico $: \dot{q}_{i}=\omega_{i}$ cuyas frbitas son densas. La primera referentia a las funciones quabi-pexibdicas puede encontrar se en la teorfa clasica del movimiento de los astros a base de 
eoiciclos desarrollada ya por los jatronomos babilonjcos. Una aplicacion del teorema KAM fue dada en 1962 por Leonto vich: En el problema plano circular restringido de 3 cuerpos. si los puntos triangulares de equilibrio relativo son estables por la parte ineal (10 que ocurre si $\mu<\frac{1}{2}(1-\sqrt{23 / 27})$ ) 10 son tambien (con la posible exclusion un no finito de valores de las masas) al considerar la parte nolineal.

Arnol'd aplico su teorema al sistema solar con el siguien te resultado: si las masas, excentricidades e inclinacio-: nes de los planetas son suficientemente pequehos, para la mayor parte de condiciones iniciales, en el sentido de medida de Lebesgue, el sistema solar es estable.

Destaquenos sin embargo que $M$. Hénon ha obtenido cotas de lo que significa en este caso "suficientemente pequeño", hallando que dichos valores deberían ser $0\left(10^{-50}\right)$ como mucho..

Sin embargo podemos citar aqus la curiosa experiencia de P. Nacozy: Si se integra numéricamente el movimiento alrede dor del sol de Júpiter y Saturno con sus masas actuales tno despreciables) duxante un perfodo largo de tiempo, no hay evolución apreciable.

Si se incrementan las masas y se repite la experiencia se halia idéntico resultado hasta que las masas son 29 veces las actuales. Entonces Saturno es expulsado rápidamente (en unos pocos centenares de arros). Parece pues que las cotas para las que es válida la teoría de KAM pueden ser notable mente aumentadas. 
Finalmente destaquemos que $H=$ cte." da unä variedad $2 n-I$ dimensional y los toros invariantes de un sistema proximo a integra ble son n-dimensionales. Si $n=2$ dichos toros "separan" la varie dad de energía constante. Si un movimiento se inicia en un toro invariante queda siempre sobre él y si entre 2 toros no puede escapar del recinto que delimitan.

Esto no es así si $n \geq 3$ en cuyo caso los toros "no separan" y el sistema puede evolucionar destruyéndase la estabilidad. Un ejemplo fue dado por Arnol'd mediante las Ilamadas cadenas de transicion de toros bigotudos. Se tiene así la llamada inestabilidad topol6gica o difusion de Arnol'd.

Segun Moser podemos imaginar el espacio fásico como una esponja estable. Los minúsculos agujeros corresponden a las zonas situadas fuera de los toros invariantes y la inestabilidad corres ponde a la existencia de finísimos tubos comunicando los agujeros.

Hasta la actualidad no se sabe si la difugion de Arnol'd se presenta en la mayoría de los sistemas hamiltonianos o si ocurre lo contrario. En todo caso es muy diffcil de determinar numéricamente.

\$6.- Colision triple: Importancia Efsica. Tearema de siegel. Regularizacion.

Otra característica esencialmente distinta del problema de 3 cuerpos respecto al de 2 es la posibilidad de colisiones triples. Se sabe (Siegel 1941) que las frbitas que conducen a colision triple forman una variedad 13-dimensional en el espacio de fases 18-dimensional. Sin embargo se cree que el conjunto de $6 r-$ bitas que pasan cerca de la coli son the tiene considerable importancia en la evolución del problena de tres cuerpos. De hecho pare ce comprobado que gran parte de las expulsiones de estrellas de una galaxia se debe a aproximacion de una estrella a una binaria 
fuerte (eg decir, formada por estrellas muy cercanas, con elevaßa energia potencial). Se esta asf en una situación proxima a colision triple.

Un resultado clásico asegura que cuando $K$ cuerpos se acercan $a$ un punto en el que colisionan, aus posiciones relativas a dicho pun to tienden a una conflguracion homotetica. Asl. en el caso $\mathrm{K}=3$ los cuerpos tienden a formar triángulo equilatero (independiente de sus masas) o a estar alineados. Un caso parecico se tiene cuando los cuerpos se alejan unos de otros en una expansion parabolica o hiper b61ica.

Siegel probo que con la excepcion de un conjunto discreto de valores de las masas las coordenadas de los tres cuerpos que colisionan (referidos aI c.d.m.) se expresan analsticamente (para $t>0$ ) en funcion de las variables $t^{1 / 2}, t^{-\lambda_{3}}, t^{-\lambda_{3}}$ en el caso equilateral $y t^{2 / 3}, t^{-\lambda_{2}}$ en el colineal. Exceptuando un conjunto discreto de masas, $\lambda_{2} \cdot \lambda_{3}$ son irracionales $y$ por tanto la singularidad para $t=0$ es esencial (a diferencia de la colision binaria, en la que el parametro de uniformizacion es $t^{2 / 3}$ ).

Asi pues, no parece que sea posible una regularizacion analftica de la colision triple en el caso general. De hecho McGehee, Mar chal $y$ Waldvogel hen dado ejemplos de casos en los que tal regularizacion es imposible.

Una reqularización más debil que la anelitica es la geometrica. Sea $\left\{x_{i}, \dot{x}_{i}\right\}$ una sucesion de condiciones iniciales que tiende hacia una c.i. $(\bar{x}, \dot{\bar{x}})$ que lleva a colision triple. si $\forall t \in R_{+}$fijo, $x\left(t, x_{i}\right)$ depende continuamente de $x_{i}$, podemos definir la prolongacion de $x(t)$ en el caso de colision triple como $\lim _{i \rightarrow \infty} x\left(t, x_{i}\right)$. Sin embargo hay ejemplos en los que $\forall \varepsilon>0$ la $\varepsilon$-bola de $(\overline{\mathbf{x}}, \dot{\bar{x}})$ contiene puntos cuyas trayectorias se alejan despues del paso por las proximidades de colision triple y uno de los cuerpos puede salir de eata gituacion con energia arbitrariamente grande. Sin embargo este es otro campo en el que hay mucho camino por recorrer. 


\section{7.- Evolucion final: Clasificacion de tipos de movimientos.}

Chazy (1922) clasifico los tipos de movimientos del problema de tres cuerpos cuando $t \rightarrow \infty$ en 7 tipos:

1) H : Movimiento hiperbolico, en que las distancias mutuas $\left|x_{i}-x_{j}\right|$ son de la forma $o(t)$.

2) $H P_{i}$ : Movimiento hiperbolico-parabolico. EI cuerpo i se aleja hiperbolicamente de los otros 2 , y estos se hallan en un movimiento parabolico lesto es la distancia es $\left.O\left(t^{2 / 3}\right)\right)$

3) $P$ : Movimiento triparab6lico: $\left|x_{i}-x_{j}\right|=0\left(t^{2 / 3}\right)$.

4) $\mathrm{HE}_{i}$ : Movimiento hiperb6lico-elsptico. Anslogo a HP pero hallándose los 2 cuerpos restantes en movimiento elsptico.

5) $P E_{i}$ : Parabolico elfptico.

6) I : Movikientos estables a la Lagange : $\sup _{i, j}\left\{\mathbb{0}_{i}-\mathrm{x}_{j} \mid\right\}<\infty$

7) os : Movimientos oscilatorios: $\underset{t \rightarrow \infty}{\lim } \sup \left\{\mid x_{i}-x_{j}\right\}=\infty$, ${\underset{l i m}{t \rightarrow \infty}}_{i, j} \sup _{i, j}\left\{\left|x_{i}-x_{j}\right|\right\}<\infty$. Del tipo 7) Chazy no disponfa de ningun ejemplo. El primero fue suministxado por sitnikov (1959). (Ver $\oint 8)$.

$S i h>0$ solo son posibles 1),2),4). Dado que $i$ puede ser 1 , 2,3 , hay un total de 7 posibles evoluciones finales para $t \rightarrow+\infty$. Las mismag se tienen para $t \rightarrow-\infty$. Puede pasarse de cualquiera de ellas para $t \rightarrow-\infty$ a cualquier otra $t \rightarrow+\infty$, segun ha siob probado por Birkhoff. Sitnikov y Alexeyev, y el conjunto de condiciones que permite el paso de una a otra tiene medida positiva (excepto en el tipo HP, cuya medica total es nula). Es decir, puede darse captura parcial $\left(\mathrm{H}_{-\infty} \rightarrow \mathrm{HE}_{\mathrm{i}+\infty}\right)$ Y su recfproco (desintegracion completa), asi como intercambio $\left(\mathrm{HE}_{\mathrm{j}-\infty} \rightarrow \mathrm{HE}_{\mathrm{j}+\infty}\right)$. Si $\mathrm{h}<0$ la situación ea mucho mas complicada. Dejando de lado el caso PE 
(de medida nula) consideramos el $\mathrm{HE}_{i}$, L $Y$ OS (cuya medida no se conoce aun\}.

Se sabe que es posible el paso de cualquiera de estas situaciones para $t \rightarrow-\infty$ a otra $t \rightarrow+\infty$. pero que Ias transiciones $H E_{i-\infty} \rightarrow \mathrm{HE}_{i+\infty} \mathrm{HE}_{j-\infty} \rightarrow \mathrm{HE}_{j+\infty} \mathrm{L}_{-\infty} \rightarrow \mathrm{I}_{+\infty}$ ocurren para conjuntos de c.i. de medida positiva mientras que los pasos $\mathrm{HE} E_{i-\infty} \rightarrow \mathrm{L}_{+\infty}, \mathrm{HE}_{i-\infty} \rightarrow \mathrm{OS}_{+\infty}$ tienen lugar para conjuntos de medida nula. Existe multitud de criterios para reconocer que se esta en un caso $\mathrm{HE}_{i}$ cuando se procede a la integracion numerica de Ias ecuaciones del moviniento.

$\mathrm{HE}_{i}$ son abiextos conexos en el espacio fásico, pero cada uno de elios tiene ramificaciones complicadas en el espacio de fases. Birkhoff decia que $\mathrm{HE}_{i}$ eran como tres corrientes de agua proviniendo de $-\infty$ que se ramificaban en un conjunto numerable de arro yos que posteriormente se juntaban para (a excepcion de una cantidad de agua de medida nula que puede quedar en $\mathrm{b}$ o os) pasar a formar Ias 3 corrientes $\mathrm{HE}_{i+\infty}$

Szebehely ha conjeturado que si la relacion entre 2 masas pequeflas es suficientemente cercana a 1 y si $c^{2} h<0$ y suficientemente cercano a cero, casi todas las soluciones son del tipo $\mathrm{HE}_{i}$ tanto para $t \rightarrow+\infty$ como para $t \rightarrow-\infty$.

\section{B. - Movimiento quasi-a Ieatorio: Problema de Sitnikov.}

Consideremos dos magas $\mathrm{m}_{1}=\mathrm{m}_{2}=1 / 2$ en movimiento el1ptico en el plano $x, y$ y una tercera masa $m_{3} \simeq 0$ en el eje $z$, pasando por el c.d.m. de $m_{1} y m_{2}$. Sea $2 \pi$ el perfodo de $m_{1} y m_{2}$. La ecuacion que rige el movimiento de $m_{3}$ es $\ddot{z}=-z\left(z^{2}+r^{2}(t)\right)^{-3 / 2}$, donde $r(t)$ es la distancia de $m_{1}$ al origen. Si la brbita es circular el movimiento de $\mathrm{m}_{3}$ es peri6aico. 
Sean $t_{k}, k \in z$, ceros de $z$. Suponemos $t_{k}<t_{k+1}$, e introducimos $s_{k}=\left[\frac{t_{k+1}-t_{k}}{2 \pi}\right]$. A cada solucion $z(t)$ podemos asociar la suce sion (infinita por ambos lados) $\left(\mathrm{s}_{\mathbf{k}}\right)$.

Teorema. (Sitnikov) Dada una excentricidad e>0 suficientemente pequena existe unentero $m=m(e)$ tal que cualquier sucesion $\left\{s_{k}\right\}$ con $s_{K} \geq$ m corresponde a una solucion de la ecuación diferencial anterior.

El teorema puede extenderse a valores de $\mathrm{m}_{3}$ no nulos $y$ de e cualesquiera (con excepcion de un conjunto discreto).

$\mathrm{Si}$, por ejemplo $\mathrm{s}_{\mathrm{k} \rightarrow \infty}$ para $\mathrm{k} \rightarrow \infty$. $\mathrm{S}_{\mathrm{k}}$ acotado para $\mathrm{k} \rightarrow-\infty$ tenaremos una transición de movimiento tipo Lagrange a oscilatorio. $S i S_{k}$ vale infinito para un valor de $k$ finito. se tendrá un escape. Aparentemente el movimiento, siendo determinista, tiene un comportamiento aleatorio ya que la sucesion $\left(s_{k}\right)$ puede ser cualquiera $\left(\cos S_{k} \geq m\right)$.

Basicamente la demostración del teorema usa el shift de Bernoulli: Sea $A$ un alfabeto finito o numerable. Considera el espacio $x$ de las sucesiones doblemente infinitas $\left\{S_{k}\right\}, S_{k}$ \&. En aicho espacio se introduce una topología defindondo como base de entornos de $s^{*}=\left\{s_{k}^{*}\right\} a, U_{j}=\left\{s\left|s_{k}=s_{k}^{*},\right| k \mid<j\right\}$. si $p$ es una medida en $\mathrm{A} \mid \sum_{\mathrm{S}_{\mathrm{K}} \mathrm{P}}\left(\mathrm{S}_{\mathrm{k}}\right)=1$, puede dotarse a $\mathrm{X}$ de la medida producto de la forma habitual. La aplicacion $\sigma: x \rightarrow x \mid(\sigma(s))_{k}=s_{k-1}$ se llama el shift de Bernoulii. Trivialmente preserva medida, es ergodica y además es un homeomorfismo. Definiendo la entropía como $\sum_{S_{k} \varepsilon_{A}}-P\left(S_{k}\right)$ In $P\left(S_{k}\right)$ se tiene que dos shiftg son isomorfos si y s6lo si tienen la misma entropía.

En el caso de que A sea finito 0 equivale a la llamada transfor mación del panadero.

La idea esencial es incluir el shift como subsistema de un homeo morfismo $\$: Y \rightarrow Y$, esto es: 
conmuta, siendo

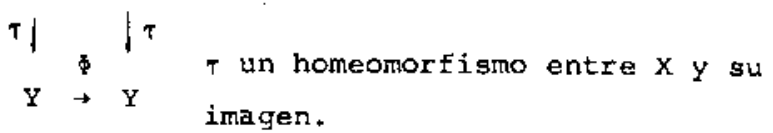

La teorla de los sistemas dinamicos quasi-aleatorios (m sistemas con entropla topologica positiva) ha sido desarrollada por A lexeyev.

Se sabe (Markus, Meyer) que con caracter generico (una propiedad es gentrica si es cierta para una intersección numerable de abiertos densos), los sistemag hamiltonianos no son ni integrables $\mathrm{nj}$ ergodicos (en cada variedad $\mathrm{H}=$ cte). Podemos conjeturar sin embargo que un comportamiento del tipo del problema de sitnikov (relacionados con Ia difusion de Arnol'd) si es generico.

\section{9. - Particularidades del problema de cuatro cuerpos: P.e.r. y} evolución final.

El problema de cuatro cuerpos ofrece un nuevo salto en cuan to a dificultad respecto al de 3 cuerpos. Sin embargo parece que no haya ya variación cualitativa de las situaciones que pue den darse al aumentar el numero de cuerpos (aunque no hay resultados en este sentido).

En cuanto a p.e.r. se conocian clásicamente algunas solucio nes. Si las masas son iguales las soluciones eran del tipo cuadrado, triángulo equilatero con una masa enel centro o colineales. El autor, en colaboración con J. Llibre, ha encontrado recientemente otra familia de soluciones en las que tres de las masas se hallan en los vertices de un triángulo isosceles y la cuarta en el interior. En total se conocen 50 p.e.r.y ge tenran fundados motivos para creer que eran las anicas. Veanse sin embargo resultados de Palmore. Smale conjeturo que la energfa potencial sobre $\frac{1}{2} \sum m_{i}\left(z_{i}\right)^{2}=1$ es función de Morge. En este caso $y$ dado que dicho conjunto es homeoformo a $\mathrm{P}_{n-2}(\mathbb{C})-\mathrm{D}(\mathrm{D}=$ colisjones $)$, la conjetura implica que el no de p.e.r. es finito para todo n. $y$ que para $n=4$ la suma al- 
ternaāa del no de p.e.r. que tienen índice por e impar es 2 (valor de la caracteristica de Euler-poincaré de $\left.P_{2}(\mathbb{C})-D\right)$. Esto se cumple en nuestro caso ya que hay 12 p.e.r. alineadas y 8 de tipo equilatero con índice 2, 24 de tipo isbsceles con indice 3 y 6 de tipo cuadrado con Indice 4.

A diferencia del caso $n=3$ en que enumero de las p.e.r. es independiente de las masas, sabemos que si una de las masas es suficien temente pequeña hay 38 p.e.r. si las otras 3 son suficientemente parecidas y 34 en caso contrario. Para los valores en que ocurre la transicion lque forman una variedad de codim. I en el espacio de lag masas) el potencial restringido deja de ser f, de Morse. Un anáisis completo esta en estudio.

Respecto a evolucion final aparecen dos tipos nuevos de comportamiento:

a) La expansion super-hiperbolica. S6lo' se presenta si $n \geq 4$. si $d(t)$ es el diametro del sistema, se tiene $d(t) / t \rightarrow \infty$. Como minimo hay 2 distancias mutuas $\left|x_{j}-x_{j}\right|$ tales que $\overline{\lim }\left|x_{i}-x_{j}\right| t_{t}=+\infty$ Iim $\left|x_{i}-x_{j}\right| / t=0$.

b) La expansión infinita en tiempo finito (Mather-McGehee). solo ge presenta si $n \geq 4$. Para ello imaginemos un cuerpo $c$ de masa peque fia oscilanco entre una binaria AB y el cuarto cuerpo D que se alejan de forma aproximadamente lineal del c.d.m.

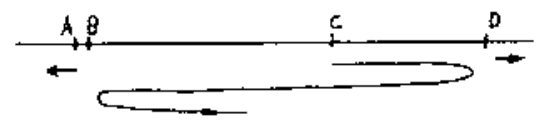

Las colisiones con $D$ son de tipo elestico. Con la binaria AB son de tipo explosivo, extrayendo $C$ tante energía como se quiera, que posteriormente comunicara a D y colapsando la binaria hacia energras potenciales mayores.

Tanto en el caso a) como en b) la demostración se lleva a cabo por un proceso andlogo al del T. de Sitnikov.

un notable esfuerzo se dedica altimamente a la obtencion de 
6rbita peribaicas del problema general de 4 cuerpos (e incluso pa ra mayor número de cuerpos) por parte de Hadjidemetrias y su escue 1a. Partiendo de un problema con 2 cuerpos no nulos y los restanteg nulos talesque las masas nulas describen orbitas peribdicas del pro blema restringido con periodos commensurables, se usa el metodo $\mathbf{E}$ h con tinuación analftica prolongando la familia de orbitas peribaicas a masas no nulas.

\$10. - Valores mayores de n. Soluciones particulares. Teorema de Moulton. Experiencias numéricas. Caso $n<1000$. Reqularizacion. Caso $\pi \geq 1000$

Para valores de $n>4$ se sabe que el problema de $n$ cuerpos puede descomponerse en subsistemas 0 cbmulos. Si cuando $t \rightarrow \infty$ esos sub sistemas se alejan unos de otros en movimiento del tipo parabolico o hiperb6Iico los centros de masas de cada subsistema tienden hacia una solucion homotetica. Poco se sabe del movimiento interno en cada cumulo (excepto si está formado por 2 o 3 cuerpos o si su movimiento ea del tipo i).

Para $n>4$ y masas cualesquiera se dispone de algunas experien cias en la busqueda de orbitas peribdicas (ver $\oint 9$ ). Si todas las ma sas excepto una son pequeñas (caso planetario) es posible la aplicación del teorema KAM (ver $(5)$ ).

Se desconoce el no y forma de los p.e.r. (ver l $^{4}$ ). Sin embargo sí se conocen para el caso alineado. En efecto. Moulton probo que en el problema de $n$ cuerpos existen paxa todos los valores de las masas n!1/2 p.e.r. alineadas. El teorema se demustra por induccion respecto al no de masas y por prolongacion respecto al valor de la Gltima masa introducida.

Por lo que antecede solo queda el reçurso de las experiencias numericas. Este ha sido utilizado in extenso $Y$ ha contribuido en gran parte al refinamiento de los metodos de resolución de e.d.o. 
Son corrientes los programas que integran las ecuaciones del movimiento para varios cientos de cuerpos, sea directamente o por métodos de Montecarlo. En todo caso debe pensarse que la integracion directa requiere pasos variables para cada cuerpo. En caso contrario cada paso de integración significaria la evaluación de $\left(\begin{array}{l}n \\ 2\end{array}\right)$ distancias. En este problema jueganun papel preponderante las binarias. Estas exigen un paso de integración muy pequeño $y$ en las proximidades de una colisión pueden tenerse errores notables. Por ello es imprescindible efectuar un paso a variables regularizadas para tratar este problema.

Una técnica debida a Heggie permite la regularización de todas las colisiones binarias. Para ello se consideran $y_{i j}=x_{i}-x_{j}$ como variables independientes $y$ se efectua la regularización ks para cada una de ellas. Sin embargo el no de grados de libertad crece de $3 n$ a $2 n(n-1)$, por lo que deja de ser practico si $n>4$. Indique mos que en este caso el Hamiltoniano es polinomial. Para valores de n mayores debe recurrirse a procedimientos aproximados mas groseros. Una técnica adecuada puede ser la de estimación del campo creado por las particulas alejadas y el estudio correcto solo para los más proximos. Otro metodo consiste en la aproximación de los terminos $\frac{1}{r_{i j}{ }^{2}}$ por cantidades greviamente almacenades en memoria. Esta técnica ha sido usada por Hohl y le permite tratar cientos de mi les de particulas. De esta manera puede conseguirse simular el com portamiento de galaxias. Sin embargo el movimiento es inestable $y$ el escape de estrellas es continuo.

\section{$\$ 11 .-$ Técnicas de perturbaciones. Teorlas planetarias. Movimientos} de la luna. Pequefos denominadores. Resanancias.

En los problemas prácticos de calculo de efemérides de astros 
del sistema solar se recure a la integracion numerica y a ks teorlas de perturbaciones. En todo caso si la orbita real se aleja lentamen te de una orbita Kepleriana, puede tomarse esta como orbita de referencia $y$. dar solo las desviaciones con respecto a la misma. En ciertos cagos es más interesante tomar como frbita de referencia otras soluciones, sean las del problema de los dos centros (como en la teorla de vinti de un satélite artificial en el campo terres tre) o las 6rbitas intermedias de Garfinkel o Aksnes para el mismo problema, o bien la 6rbita de variacion de Hill para el movimiento de la luna, etc.

otra tecnica consiste en facilitar en cada momento la orbita Kepleriana ( o no) ogculatriz. Este se define como la 6rbita keple riana cuya posicion y velocidad coinciden en cada momento con la orbita real. Por supuesto que los elementos $a, e, I, w, \Omega, M_{0}-t^{-1 / 3}$. serán funciones del tiempo. Sea $\sigma$ el vector formado por dichos elementos. Se tendrá $\sigma=\varepsilon \varphi(\sigma, t)$, donde el factor $\varepsilon$ indica que ó serf́a cerosi no hubiera la perturbación, $Y$ que ésta se supone pequefia. Partiendo de un valor inicial $\sigma_{n}$ se obtiene $\sigma_{4}$ median te $\sigma_{1}=\sigma_{0}+c \int_{0}^{k} \oplus\left(\sigma_{0}, \tau\right) d_{T}$. El método de Picara deja de ser prác tico ya gue puede $\phi\left(\sigma_{1}, t\right)$ ser expresion diffeil. Se aplica un desallo de Taylor limitado $\varphi\left(\sigma_{1}, t\right)=\varphi\left(\sigma_{0}, t\right)+D \varphi\left(\sigma_{0}, t\right) \varepsilon \int_{0}^{t} \varphi\left(\sigma_{0}, \tau\right) d \tau$ $\operatorname{con} 10$ que $\sigma_{2}=\sigma_{1}+e^{2} \int_{0}^{t} D \varphi\left(\sigma_{0}, \tau\right) \int_{0}^{\tau} \varphi\left(\sigma_{0}, \xi\right) d \xi$.

La iteración da lugar al método clasico de teorfa de perturbaciones iniciado por Lagrange, Laplace y Poisson.

Los métodos de series de Lie, promedios, von Zeipel, etc. consisten en técnicas iterativas para eliminar la perturbacion del hamiltoniano inicial $\mathrm{H}=\mathrm{H}_{0}+\mathrm{a}_{1}+\mathrm{q}^{2} \mathrm{H}_{2}+\ldots$ con objeto de convertir-, lo en un hamiltoniano independiente de las variables angulares.

Las sucesivas iteraciones $y$ su convergencia se ven dificultadas 
por la aparicion. de pequeños denominadores. Por ejemplo, en el mé todo cláaico se tiene en cada paso que la funcion a integrar respecto al tiempo es del tipo $\Sigma \quad A_{\mathbf{p}}(p)$ exp $i(k, q)$ y si $(k, \dot{q})$ es $k \in z^{3}$

pequero dicho térnino aparece multiplicado por un factor grande. Este hecho implica que la mayor parte de las series utilizadas en mecśnica celeste tienen caracter asintotico $y$ no son validas uniEormemente.

En las teorfas planetarias se suele usar como pequeño parametro

la masa de los cuerpos perturbadores. El estudio iniciado por Laplace de la variación de e $Y$ w para los distintos planetas debi do a sus perturbaciones (aI menos en una aproximacion de primer or den) conaujo a introducir la ecuacion o polinomio caracter śtico.

La teoría de la luna es un ejemplo de la dificultad que encierran los calculos en Mecánica celeste. Por una parte ni su masa ni la perturbacion solar son pequenas, con lo que los desarrollos que se obtienen convergen lentamente. Por otra parte la precision de las medidas actuales (del orden de decenas de cm. en la distancia mediante uso de reflectores laser) obliga a utilizar teor 1 as de or den muy elevado. A titulo de ejemplo digaros que una teoria aceptable de la luna que diera correctamente su posicion con error de unos pocos metros por siglo. requeriria tener un cuenta la influencia de todos los planetas (excepto pluton) y trabajar con desarrollos de Fourier conteniendo miliones de términos.

Un tal trabajo requiere el uso de ordenadores en el manejo de expresiones analfticas y en procesos analiticos de derivacion, inte gracion y multiplicacion de series.

El ajuste de las desviaciones de la posicion de la luna respeç to a las que predice la teorla newtoniana mediante precisas y muy numerosas obsexvaciones de ocultaciones de estrellas por la misma ha permitido constatar la disminución de la cons- 
tante de la gravitacion y en breve ge dispondra de valores de la velocidad de variacion.

Finelmente indiquemos que la aparicion de pequeños denominadoroo esta relacionada con el fenbmenc ie resonancia. Hay que destacar las numerosas resonancias que se producen en el sistema solar, tanto entre planetas como con satelites, asteroides, o con anillos de SAturno. Los asteroides evitan ciertos valores del movimiento medio mientras que muestran preferencia por otros. Se esta lejos de tener una explicacion satisfactoria de estos hechos, pero en to do caso la resonancia parece tener un papel importante en la evolucion del sistema solar hacia su estado actual.

CONCIUSION: Wintner decia que toda generacion de matematicos ataca el problema de 3 cuerpos ( $y$ por extension el de $n$ ) de una manera peculiar y con herramientas propias. Hoy en dia podemos afirnar que métodos utilizados abarcan de la topologfa al estudio probabilfstico, de la teorfa de puntos crfticos a las aproximaciones diofanti cas $y$ del analisis no lineal a las técnicas numéricas. Además la precision alcanzada por la tecnica en las mediciones ha permitido que las medidas sean mucho mas precisas que las teorfas, obligando a rehacerlas y a estudiar las bases Exsicas en que se apoyan. Concluyamos con una anecdota。 v. Szebehely, autor del mejor texto sobre el problema restringido $y$ de numerosfsimos art y persona sumamente afable, ha felicitado las Navidades del 75 a sus amigos $y$ conocidos que trabajan en Mecanica Celeste diciendo: Deseo fervientemente que usted "resuelva" en 1976 el problema de 3 cuerpos. 


\section{BIBLIOGRAFIA}

Abraham, R., Marsden,J.E.: Foundations of Mechanics, Benjamin, 1967 Alexeyev,V.M.: "Quasirandom dynamical systems. I, II, III", Math. USSR Sbornik, $5,(1968), 73-128 ; 6,(1968), 505-560 ; \underline{7},(1959)$ 1-43.

Alexeyev,V.M.:" Sur l'allure finale du mouvement dans le probleme des trois corps", Actes Congres Inter. Math., Nice, 1970, Tome 2, 893-907, Gauthier-viliars.

Arnol'd,v.I.: "Small denominators and problems of stability of motion in classical and Celestial Mechanics", Russ. Math. Surveys, 18, (1963), 85-192.

Arnol'd,V.I.: "Instability of Dynamical Systems with Several Degrees of Freedom", Soviet Math. Dokl., ․, (1964), 581-585. Arnol'd,v.I., Avez,A.: Ergodic Problems of Classical Mechanics. Benjamin, 1968.

Bartlett,J.H.: "The Reatricted Problem of Three Bodies, I.II", Mat. Fys. Skr. Vid. Selsk., 2․ no 7, (1964); ‥ no 1, (1965).

Birkhoff.G.: Dynamical Systems, Am. Math. Soc., I927.

Cabral, H.E.: "On the Integral Manifolds of the N-Body Problem", Inventiones Math., 20, (1973) 50-72.

Chazy.J.: "Sur I'allure finale du mouvement dans le probleme des 3 corps quand le temps crôt indefiniment". Annales de I'Ecole Normale Superieure, $3^{\mathrm{e}}$ serie, 39, (1922) 29-130. Easton, R. "The Topology of the Regularized Integral Surfaces of the 3-Body Problem", J. of Diff. Equa., 12. (1972) 361384.

Easton, R.: "Some Topology of n-Body Problems", J. of Diff. Equa., 19. (1975) 258-269.

Escobal, P.R.: Methods of Orbit Determination, J.Wiley, 1965. 
Gaugs,K.F.: Theoria Motug, Dover (reprint) 1963

Hadjidenetriou, J.D.: "Familieg of Periodic orbits in the General

N-Body Problem", en Long-Time Prediction in Dynamics ; 223-240, Ed. V. Szebehely, B.D. Tapley, Reidel, 1976.

Hagihara, Y.: Celestial Mechanics, Vol. 1, MIT Press, 1970; Vol.

2, MIT Press, 1972; Vol. 3, Japan Society for the Pro motion of the Science, 1974; Vol. 4, Japan Soc., 1975.

Heggie.D.: "A Global Regularisation of the Gravitational N-Body Problem", Celestial Mechanics, 20, (1974) 217-242.

Hénon, M.: "Exploration numerique du probleme restreint. III, IV", Bulletin Astrononique, serie 3, 1, fasc. 1, 57, (1966), fasc. 2, 49 (1966)

Hohl,F.: "N-Body Simulations of disks". en Dynamics of Stellar

Systems, A. Hayli (editor), IAU Symposium, no 69,

P. 349, Reidel, 1975.

Kolmogoroff. A.N.: "General Theory of Dynamical Systems and classical Mechanics", Proceed. 1954, Inter. Cong. Math fen ruso). Traducido al inglés en Abraham, apéndice D.

Leontovich, A.M.: "On the Stability of the Lagrange pexiodic Solum tions for the Reduced Problem of the Three Bodies". Soviet Math. Doklady, $\underline{3}$, (1965) 425-430.

Losco, L.: "Sur un invariant integral du probleme des n-corpg: consequence de l'homogeneite du potentiel". en The Stability of the Solar System and of Small stellar Sys-i tems. Y. KOzai (Editor), IAU Symposium No 62, 249-255, Reidel, 1974.

McGehee, R.: "Triple collision in the collinear Three-Body Pro- blem". Inventiones Math., 27, (1974) 191-227.

McGehee, R.. Mather, J.N.: "Solutions of the collinear four body problem which become unbounded in finite time", Battelie Recontres, 1974 Proceedings.

Marchal, Ch.: "Qualitative Methods and Results in Celestial Mecha- 
nics", en Long-Time Predictions in Dynamics, Ed. V. Szebehly, B.D. Tapley, 181-208, Reidel, 1976.

Markus,L.,Meyer,K.R.: "Generic Hamiltonian Dynamical Systems are neither Integrable nor Ergodic", Memoirs of the A.M.S. . 144, 1974 .

Moser,J.: "Nonexistence of Integrals for Canonical systems of Differential Equations" Comm. on Pure and Appl.Math., 8. (1955) 409-436.

Moser. J.: "On invariant curves of area-preserving mappings of an ennulus", Nach. Akad. Wiss. Göttingen, Math. Phys. Klass., 1, (I962), 1-20.

Moser,J.: "Regularization of Kepler's Problem. and the Averaging Method on a Manizold", Comm. on Pure \& Appl. Math.. 23. (1970) 609-636.

Moser, J.: Stable and Random Motion in Dynamical Systems. Princeton Unjv. Press, 1973.

Moulton, F.R.: "The Straight Iine solutions of the problem of N-bodies", Annais of Math., 12, (1910), I-17.

Palmore, J. : "Classifying Relative Equilibria.I,II", BuII. A.M.S., 79. (1973) 904-908; 81, (1975), 489-491.

Palmore.J.: "Clasgifying Relative Equilibria, III", Letters in Math. Phys., 1. (1975) 71-73.

Poincaré, H.: Les methodes nouvelles de la Mécanique celeste. Gathier-Viliars, 1892, 1893, 199.

Robinson, C.: "Lectures on Hamiltonian Systems", Monografias de Matematica, Guanabara, 1971.

Rísmann, H.: "Woer invariante Kurven differenzierbarer Abbildungen eines kreisringes", Narichten Akid. Wiss. Gobtingen Math. Phys. Klass., 1970.

Saari, D.G.: "Expanding gravitational systens". T.A.M.S., 156. (1971) $1-22$.

SiegeI, C.I., Moser,J.K.: Lectures on Celestial Mechanics, Springer-verlag, 1971. 
simo, c.: "La variedad de orbitas keplerianas y la teorfa general de perturbaciones" Tesis, Universidad de Barcelona, 1974.

Simo, C.: "Aspects Topologiques en Mecanique celeste", Table ronde de Mec. Cel., Institut Henri Poincare, paris, 21. $\operatorname{mai} 1975$.

Simo, c.: "Posiciones de equilibrto relativo del problema de $3+1$ cuerpog $Y$ su evolucion ${ }^{2}$, Comunicacion en la Reunion Anual de Matematicos Espafoles, Malaga, 1976. Sitnikov, K.A.: "The existence of oscillatory motions in 3-body problem", Soviet Phys. Doklady, $\underline{5}$, (1961), 647-650.

Smele,S.: "Topology and Mechanics". Inventiones Math., 10, (1970)! 305-331: 11. (1970) 45-64.

Souriau,J.M.: Structure des Systernes dynamiques, Dunod, 1970

Stermberg,s.: Celestial Mechanics, Benjamin, 1969

Stiefel, E.L.. Scheifele, G.: Linear and Reqular Celestial Mechanics, Springer-verlag, 1971

Szebehely, v.: Theory of Orbits. The restricted problem of 3 bodies, Academic-Press, 1967.

Waldvogel, J.: "Triple collision" en Long-Time Predictions in Dynamics, 241-258, Ed. v. Szebehely, B.D. Tapley, Rei- ! del, 1976.

Whittaker, E.T.: A Treatise on the Analytical Dynamics of partiges and rigid bodieg, Cambridge Univ. Press, 1973.

wintner, A.: The Analytical Foundationg of Celestial Mechanics, Princeton Univ. Press, 1941. 